\title{
Pre-procedural risk models for patients undergoing transcatheter aortic valve implantation
}

\author{
Glen P. Martin ${ }^{1}$, Matthew Sperrin ${ }^{1}$, Mamas A. Mamas ${ }^{2}$ \\ ${ }^{1}$ Division of Informatics, Imaging and Data Science, Faculty of Biology, Medicine and Health, University of Manchester, Manchester Academic \\ Health Science Centre, Manchester, UK; ${ }^{2}$ Keele Cardiovascular Research Group, Centre for Prognosis Research, Institute for Primary Care and \\ Health Sciences, Keele University, Stoke-on-Trent, UK \\ Contributions: (I) Conception and design: MA Mamas; (II) Administrative support: None; (III) Provision of study materials or patients: None; (IV) \\ Collection and assembly of data: None; (V) Data analysis and interpretation: None; (VI) Manuscript writing: All authors; (VII) Final approval of \\ manuscript: All authors. \\ Correspondence to: Dr. Glen Philip Martin, PhD. Division of Informatics, Imaging and Data Science, Faculty of Biology, Medicine and Health, \\ University of Manchester, Vaughan House, Manchester, M13 9GB, UK. Email: glen.martin@manchester.ac.uk.
}

\begin{abstract}
Transcatheter aortic valve implantation (TAVI) has emerged as the standard treatment option for patients with symptomatic aortic stenosis who are considered intermediate to high surgical risk. Nonetheless, optimal clinical outcomes following the procedure require careful consideration of procedural risk by the Heart Team. While this decision-making could be supported through the development of TAVIspecific clinical prediction models (CPMs), current models remain suboptimal. In this review paper, we aimed to outline the performance of several recently derived TAVI CPMs that predict mortality and present some future research directions. We discuss how the existing risk models have achieved only moderate discrimination but highlight that some of the models are well calibrated across multiple populations, indicating the feasibility of using them to aid benchmarking analyses. Moreover, we suggest that future work should focus on the development of CPMs in cohorts of patients with aortic stenosis that include multiple treatment modalities. Supported by appropriate modelling of 'what if' scenarios, this would allow the Heart Teams to predict and compare outcomes across surgical aortic valve replacement, medical management and TAVI, thereby allowing one to personalise treatment decisions to the individual patient. Such a goal could be facilitated by considering novel risk factors, shifting the focus to endpoints other than mortality, and through collaborative efforts to combine the evidence base and existing models across wider populations.
\end{abstract}

Keywords: Transcatheter aortic valve implantation (TAVI); aortic stenosis; clinical prediction models (CPMs); mortality; risk assessment; surgical aortic valve replacement (SAVR)

Submitted Apr 09, 2018. Accepted for publication May 04, 2018.

doi: $10.21037 /$ jtd.2018.05.67

View this article at: http://dx.doi.org/10.21037/jtd.2018.05.67

\section{Introduction}

Symptomatic aortic stenosis is the most prevalent valve pathology $(1,2)$, with surgical aortic valve replacement (SAVR) being the mainstream treatment strategy. While SAVR has been shown to be effective $(3,4)$, a significant proportion of patients are deemed too high surgical risk, due to their age and associated comorbid burden (5). As such, transcatheter aortic valve implantation (TAVI) shifted the paradigm for treating patients with symptomatic aortic stenosis who were considered high operative risk $(6,7)$. Additionally, contemporary data have shown similar outcomes between transfemoral TAVI and SAVR for intermediate risk patients (8-10). Hence, Heart Teams need to compare the perceived benefits/risks across both treatment options. This process is based on clinical judgement on an individual patient basis, with limited tools available to aid this decision-making. Although risk 


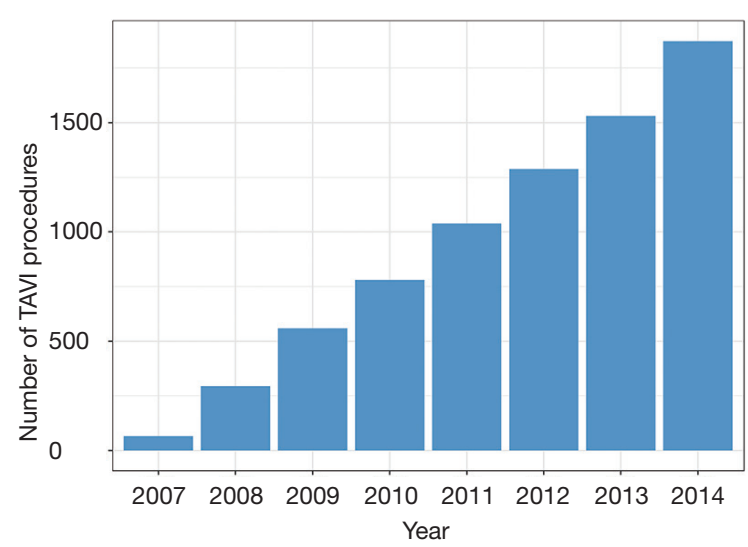

Figure 1 The annual number of TAVI procedures conducted in the UK between 2007 and 2014. TAVI, transcatheter aortic valve implantation.

stratification tools for SAVR are in clinical use, developing a systematic way of measuring procedural risks in patients considered for TAVI is a priority (11).

Mortality remains at the forefront of risk stratification following surgical procedures. To this end, clinical prediction models (CPMs) underpin pre-procedural risk assessment and can aid clinical decision-making, the consent process and benchmarking/auditing (12). Cardiac surgery CPMs, such as the EuroSCORE and Society of Thoracic Surgeons Predicted Risk of Mortality (STSPROM) models $(13,14)$, have been used to quantify patient risk in TAVI randomised controlled trials (6,7). However, these models inappropriately estimate mortality risk within TAVI cohorts, since they were derived to predict shortterm mortality within surgical populations; as such, their predictive performance is known to be poor in patients undergoing TAVI (15-17).

Therefore, estimating the risks of mortality and poor outcomes following TAVI through the derivation of TAVIspecific CPMs is an ongoing research area. The aim of this review article is to outline the current landscape of pre-procedural risk assessment in TAVI, and to propose suggestions for unmet clinical needs in this area. We begin by overviewing current risk models and their reported predictive performance, and then discuss some of the challenges facing TAVI risk assessment. We end with some suggestions for future perspectives.

\section{Risk prediction in TAVI}

The worldwide increase in the annual number of TAVI procedures has driven a multitude of national registries. For example, in the UK, the annual number of TAVI procedures increased rapidly between 2007 and 2014 (Figure 1). National registries provide a valuable platform in which to study outcomes at scale, while also facilitating risk model development. The latter is important to aid the identification of high-risk TAVI cases and for operator/ centre performance comparisons, particularly in the cascade of a new treatment where technology is rapidly developing. Indeed, there have been several recent attempts at deriving TAVI-specific CPMs from national registries, each aiming to predict the risk of either in-hospital/30-day mortality $(18-23)$ or one-year mortality $(18,24,25)$. A review of these models and their risk factors is given in Tables 1,2 for short- and long-term mortality prediction, respectively. Unsurprisingly, many of these models share similar sets of risk factors, although the definitions of each vary between the different cohorts, owing to differences in both recording practices and modelling strategies.

One of the earliest TAVI risk models was the FRANCE-2 model (22), which included nine risk factors (Table 1), contributing to a 21-point score to predict 30-day mortality, and was derived on 2,552 patients from the France TAVI registry. Similarly, the Society of Thoracic Surgeons/ American College of Cardiology Transcatheter Valve Therapy (STS/ACC) model includes a comparable collection of risk factors, and represents the CPM derived on the largest cohort of TAVI patients to-date (19). Specifically, the model was developed on 13,718 patients, and internally validated on a further 6,868 patients (Table 1 ).

In contrast, only a handful of TAVI CPMs have aimed to predict one-year mortality (Table 2). For example, the CoreValve model developed by Hermiller et al. (18) was derived and internally validated on 3,687 patients from the CoreValve US Pivotal Trial, and aimed to predict both 30-day and one-year mortality. The one-year mortality model was based on extending the STS-PROM score to include indicators of Albumin levels, Charlson score and home oxygen use (Table 2). While this represents the largest study used to develop a one-year mortality TAVI CPM todate, the applicability to patients not undergoing TAVI with a CoreValve prosthesis remains unclear.

\section{Performance of existing risk prediction models}

Despite the emergence of several TAVI CPMs, one of the main barriers to implementing them in clinical practice is the paucity of external validation studies that have examined 
Table 1 Risk factors included across the existing TAVI-specific CPMs that aim to predict in-hospital and/or 30-day mortality

\begin{tabular}{|c|c|c|c|c|c|}
\hline Characteristic & CoreValve model (18) & STS/ACC (19) & OBSERVANT (21) & FRANCE-2 (22) & UK-TAVI (23) \\
\hline Total sample size & 3,687 & 20,586 & 1,878 & 3,833 & 6,339 \\
\hline Derivation & 2,482 & 13,718 & 1,256 & 2,552 & 6,339 \\
\hline Validation* & 1,205 & 6,868 & 622 & 1,281 & $6,339^{\dagger}$ \\
\hline \multirow{11}{*}{ Predictors } & Assisted living & eGFR & Critical state & $\mathrm{BMI}<30 \mathrm{~kg} / \mathrm{m}^{2}$ & Female \\
\hline & Home oxygen & Dialysis & Pulmonary & NYHA class IV & BMI \\
\hline & Age $>85$ years & NYHA class IV & Hypertension & $\geq 2$ pulmonary oedemas & eGFR \\
\hline & & Severe chronic lung disease & Diabetes & Pulmonary hypertension & Pulmonary disease \\
\hline & & & LVEF $<40 \%$ & Dialysis & Prior BAV \\
\hline & & & & Nonfemoral access & Critical state \\
\hline & & & & & Poor mobility \\
\hline & & & & & KATZ \\
\hline & & & & & PA systolic pressure \\
\hline & & & & & Non-elective procedure \\
\hline & & & & & Nonfemoral access \\
\hline
\end{tabular}

*, Internal validation; $\dagger$, obtained by bootstrap resampling. TAVI, transcatheter aortic valve implantation; CPMs, clinical prediction models; BAV, balloon aortic valvuloplasty; BMI, body mass index; eGFR, Glomerular filtration rate; KATZ, index of independence in daily living; LVEF, left ventricular ejection fraction; NYHA, New York Heart Association.

their predictive performance in populations distinct to those where they were developed (26-28). Early examples of external validation studies were those conducted by Halkin et al. using the Israeli TAVI registry (29), and by our group within the UK TAVI registry (17), with both finding that the existing TAVI CPMs had only moderate predictive performance. For example, the investigation from the UK TAVI registry compared the performance of the German AV (20), the FRANCE-2 (22), the OBSERVANT (21) and the STS/ACC (19) CPMs with the surgical risk models, with the $\mathrm{C}$-statistics ranging from 0.57 (OBSERVANT) to 0.64 (STS/ACC) (17). These results were later supported within the Swiss TAVI registry, where Pilgrim et al. externally validated the STS/ACC CPM on 3,491 patients and found that the STS/ACC model was well-calibrated but achieved only moderate discrimination (C-statistic of 0.66) (30). Subsequently, the STS/ACC model was shown to have a C-statistic of 0.68 when applied to patients from a single centre in Germany (31).

As such, the emerging evidence suggests that the TAVI models perform better than their surgical counterparts do, albeit with moderate discrimination. Moreover, the current evidence suggests that the models could support clinical benchmarking and auditing, as proposed by the STS/ACC model (19) and the UK-TAVI model (23). In particular, the STS/ACC model has been externally validated most widely, with each study showing appropriate calibration (17,30,31). Nevertheless, further external validation studies are required to assess the performance of all existing TAVI CPMs in distinct populations, and the moderate discrimination inhibits their use in aiding mortality risk stratification on an individual patient basis.

\section{Challenges to mortality risk prediction in TAVI}

One potential explanation for the limited performance 
Table 2 Risk factors included across the existing TAVI-specific CPMs that aim to predict 1-year mortality

\begin{tabular}{|c|c|c|c|}
\hline Characteristic & CoreValve model (18) & TARIS (24) & TAVI2-SCOREe (25) \\
\hline Total sample size & 3,687 & 1,178 & 511 \\
\hline Derivation & 2,482 & 845 & 511 \\
\hline Validation* & 1,205 & 333 & 100 (bootstrap resampling) \\
\hline \multirow{5}{*}{ Predictors } & Home oxygen & Female & Male \\
\hline & Severe Charlson score & BMI & Porcelain aorta \\
\hline & STS-PROM > 7\% & Pulmonary hypertension & $\mathrm{Ml}<90$ days \\
\hline & & eGFR & $\mathrm{CrCl}<30 \mathrm{~mL} / \mathrm{kg} / \mathrm{min}$ \\
\hline & & LVEF $<45 \%$ & Aortic valve mean gradient $\geq 70 \mathrm{mmHg}$ \\
\hline
\end{tabular}

*, Internal validation. TAVI, transcatheter aortic valve implantation; CPMs, clinical prediction models; BMI, body mass index; CrCl, creatinine clearance; eGFR, glomerular filtration rate; LVEF, left ventricular ejection fraction; MI, myocardial infarction; STS-PROM, Society of Thoracic Surgeons Predicted Risk of Mortality.

of existing TAVI CPMs is that they have been derived in registries that predominately include elderly and highrisk surgical candidates and have mainly aimed to predict mortality. However, elderly patients are at high risk of mortality from causes unrelated to the TAVI procedure, mostly non-cardiac conditions, and previous work has demonstrated that mortality risks in the most elderly patients quickly return to that of a matched general population (32). While this is undoubtedly an artefact of the careful patient selection in the most elderly TAVI patients, it does highlight the difficulties in trying to use patient baseline characteristics to predict all-cause mortality in the long-term follow-up. Reframing the problem to predict how long it will take a patient's survival trajectory to return to that of a matched healthy individual, might prove beneficial in this setting.

Similarly, homogenous cohorts make it challenging to find novel risk factors that can discriminate between those at high- and low-risk of mortality. For example, frailty has been suggested as a potential additive marker to improve mortality prediction in TAVI, with previous studies showing frailty to be associated with TAVI outcomes (33-35). However, the lack of recording of frailty-related variables as well as its unstandardized definition, have hindered incorporating such measures into TAVI CPMs $(19,22)$. Recent studies have explored the benefit of adding frailty scores into conventional surgical CPMs $(33,34,36,37)$, with each showing a statistically significant, but modest, improvement in the predictive performance after adding the frailty variables. Hence, although frailty is widely suggested as a potential marker to improve prediction, incorporating this concept into CPMs remains a challenge. Specifically, there is no consensus around the most clinically useful measure of frailty [as exemplified by the range of measures used across the previous literature $(33,34,36,37)]$, and many of the objective measures (e.g., hand-grip, gait speed, 6-min walk distance, etc.) are not yet routinely collected in national registries or institutional datasets. As such, there is a need to enhance frailty assessment in TAVI, and to utilise alternative measures such as functional measurements, imaging parameters and serological markers. The potential expansion of TAVI into intermediate (8) and lower risk (9) patients, means the identification of such novel risk factors may become increasingly important, especially since this expansion might reduce the impact that frailty and other comorbidities have on pre-procedural risk assessment.

On the other hand, the expansion of TAVI into wider patient groups and the rapid improvements in device technology also presents an additional challenge. Indeed, the majority of CPMs that are used across clinical practice are usually regarded as static tools. However, with changes in the underlying case-mix and procedural practice, there 
should be corresponding changes to TAVI risk prediction, since CPMs should reflect most contemporary practice. Lack of updating resulted in the so-called 'calibration drift' observed in the Logistic EuroSCORE, where there was a divergence between the observed and expected outcome risks (38). While methodology for dynamic risk prediction exist $(39,40)$, these methods are rarely incorporated into contemporary risk scores. Accordingly, the rapid changes in device technology make TAVI an ideal setting in which to utilise these advanced dynamic modelling methods, to aim for improved and timely risk predictions.

\section{Future perspectives: challenging the current state of knowledge}

The existing models serve as a useful foundation in which to adjust centre level mortality outcomes for cross-study comparison and for audit analyses, but they cannot be used on an individual patient level. Given that randomised controlled trials have shown equipoise between SAVR and TAVI in intermediate risk patients at a cohort level (8), there is an increasing clinical need in differentiating expected outcomes across these treatment modalities at an individual patient level to decide optimal treatment pathways. Aortic stenosis represents an ideal clinical setting in which to do this, since successfully optimising treatment decisions between SAVR and TAVI could lead to benefits to patients, clinicians, resource utilisation and policy makers. Specifically, we are interested in comparing the predicted risks for patients assuming they undergo different interventions, and then targeting the intervention that minimises the risk of poor outcomes. However, there are both methodological and clinical hurdles that need to be tackled in this space.

From a methodological viewpoint, standard CPM development strategies are ill-placed to support decisionmaking about treatment initiation since they do not explicitly consider 'what if' scenarios (41). Additionally, such a model could only be achieved by modelling within cohorts that include all patients with severe aortic stenosis (regardless of treatment regime), rather than modelling solely within patients who have already undergone TAVI (e.g., national registries). This suggests that a more unified approach to modelling will be required that joins networks of modelling teams.

From a clinical viewpoint, comparing the risks across multiple treatment modalities also requires one to consider a range of clinical, non-clinical and patient-reported outcomes, especially in a predominately-elderly population that have multiple comorbidities, or more recently, in lower risk patients that are expected to have a longer life expectancy (42). For example, the majority of existing TAVI CPMs have aimed to predict mortality (at either 30-day or 1-year), but outcomes such as hospital readmission, general health status and improvements in quality of life are equally important considerations for patient/treatment selection. Definitions of "poor outcome" following TAVI have previously been proposed that integrate mortality and quality of life (43), with such definitions used to derive the PARTNER risk model that aimed to estimate risk of poor outcome 6-months after TAVI (44). The model achieved a moderate discrimination (C-statistic of 0.66) in development, with similar performance reported when the model was externally validated (45). By defining and predicting a "poor outcome", the PARTNER model was pivotal in advancing the ability to identify subgroups of TAVI candidates who are at high-risk of adverse outcomes, although the moderate discrimination limits the ability to do this on an individual patient basis. Nevertheless, TAVI cohorts represent a fairly unique patient population, where the relative trade-offs between improved survival and improvements in quality of life are likely to vary between patients and their personal circumstances (44). Thus, a 'one-size-fits-all' definition of poor TAVI outcomes is potentially overly simplistic. To achieve a truly personalised prediction in patients with aortic stenosis, one needs to integrate a patient's individual definition of what constitutes a 'poor outcome' and incorporate this dynamic outcome definition within the treatment decision-making; patient-reported outcomes and the Heart Teams will both play a vital role in this assessment.

Nonetheless, we must maintain perspective on striving for a risk score that differentiates expected outcomes between SAVR and TAVI. Specifically, there has been a rise in the number of publications that have aimed to develop new TAVI CPMs in new populations of interest. While this is expected without a widely agreed model, we need to guard against creating a proliferation of models, each of which are developed in silos of datasets and outcomes; this is true across most clinical contexts, as exemplified by a recent editorial (46). Arguably, since TAVI registries are generally smaller than their counterparts in cardiac surgery (such as those used to derive the EuroSCORE or STS-PROM models), there is scope to move away from standard modelling approaches and unify the evidence across populations. A similar recommendation was made 
by Puri et al. (11), who suggested a pragmatic approach to TAVI risk assessment. Here, the predicted risks from (I) the PARTNER (44) and FRANCE-2 (22) CPMs, (II) frailty indicators and (III) organ failure, where cross-tabulated in a relatively crude way to define groups of low-, intermediate-, high- and prohibitive-risk (11). However, one could achieve this in a more integrated approach, by combining the whole evidence base (i.e., all existing TAVI CPMs), new data and emerging risk factors/outcome definitions using model updating and model aggregation methods (47-50). This would allow a holistic view of TAVI risk assessment and potentially aid the identification of high-risk cases on an individual patient basis.

\section{Conclusions}

In this review, we have discussed some of the challenges facing pre-procedural risk assessment in patients undergoing TAVI. There has been positive progress in this space through the development of several TAVI-specific CPMs. Importantly, some models have shown potential to facilitate benchmarking and cross-study comparisons. However, there are still areas to improve before any of the current risk models can support pre-procedural risk assessment on an individual patient basis. The potential expansion of TAVI into younger and lower risk patients means there is a need for CPMs that differentiate between outcomes from SAVR and TAVI. Specifically, rather than modelling solely within TAVI cohorts, future studies should focus on developing CPMs within cohorts of patients with aortic stenosis that have been treated through multiple treatment strategies. Supported by appropriate consideration of 'what-if' scenarios, this would allow one to compare predicted risks across treatment modalities for the individual patient and target intervention accordingly. However, we must maintain perspective on this by carefully defining "poor outcomes" and avoid proliferation of models derived in silos of populations.

\section{Acknowledgements}

Funding: This work was partially funded by the Medical Research Council through the Health e-Research Centre, University of Manchester [MR/K006665/1].

\section{Footnote}

Conflicts of Interest: The authors have no conflicts of interest to declare.

\section{References}

1. Supino PG, Borer JS, Preibisz J, et al. The epidemiology of valvular heart disease: a growing public health problem. Heart Fail Clin 2006;2:379-93.

2. Carabello BA, Paulus WJ. Aortic stenosis. Lancet 2009;373:956-66.

3. Brown JM, O'Brien SM, Wu C, et al. Isolated aortic valve replacement in North America comprising 108,687 patients in 10 years: Changes in risks, valve types, and outcomes in the Society of Thoracic Surgeons National Database. J Thorac Cardiovasc Surg 2009;137:82-90.

4. Kvidal P, Bergström R, Hörte LGG, et al. Observed and relative survival after aortic valve replacement. J Am Coll Cardiol 2000;35:747-56.

5. Iung B, Cachier A, Baron G, et al. Decision-making in elderly patients with severe aortic stenosis: Why are so many denied surgery? Eur Heart J 2005;26:2714-20.

6. Leon MB, Smith C, Mack M, et al. Transcatheter aorticvalve implantation for aortic stenosis in patients who cannot undergo surgery. N Engl J Med 2010;363:1597-607.

7. Smith CR, Leon $M$, Mack $M$, et al. Transcatheter versus surgical aortic-valve replacement in high-risk patients. N Engl J Med 2011;364:2187-98.

8. Leon MB, Smith CR, Mack MJ, et al. Transcatheter or surgical aortic-valve replacement in intermediate-risk patients. N Engl J Med 2016;374:1609-20.

9. Abdelghani M, Serruys PW. Transcatheter aortic valve implantation in lower-risk patients with aortic stenosis. Circ Cardiovasc Interv 2016;9:e002944.

10. Thourani VH, Kodali S, Makkar RR, et al. Transcatheter aortic valve replacement versus surgical valve replacement in intermediate-risk patients: a propensity score analysis. Lancet 2016;387:2218-25.

11. Puri R, Iung B, Cohen DJ, et al. TAVI or No TAVI: identifying patients unlikely to benefit from transcatheter aortic valve implantation. Eur Heart J 2016;37:2217-25.

12. Steyerberg EW. Clinical Prediction Models. Springer New York; 2009.

13. Nashef SAM, Roques F, Sharples LD, et al. EuroSCORE II. Eur J Cardiothorac Surg 2012;41:734-44; discussion 744-5.

14. O'Brien SM, Shahian DM, Filardo G, et al. The Society of Thoracic Surgeons 2008 Cardiac Surgery Risk Models: Part 2-Isolated Valve Surgery. Ann Thorac Surg 2009;88:S23-42.

15. Ben-Dor I, Gaglia MA, Barbash IM, et al. Comparison between Society of Thoracic Surgeons score and logistic 
EuroSCORE for predicting mortality in patients referred for transcatheter aortic valve implantation. Cardiovasc Revasc Med 2011;12:345-9.

16. Piazza N, Wenaweser P, van Gameren M, et al. Relationship between the logistic EuroSCORE and the Society of Thoracic Surgeons Predicted Risk of Mortality score in patients implanted with the CoreValve ReValving system--a Bern-Rotterdam Study. Am Heart J 2010;159:323-9.

17. Martin GP, Sperrin M, Ludman PF, et al. Inadequacy of existing clinical prediction models for predicting mortality after transcatheter aortic valve implantation. Am Heart J 2017;184:97-105.

18. Hermiller JB, Yakubov SJ, Reardon MJ, et al. Predicting early and late mortality after transcatheter aortic valve replacement. J Am Coll Cardiol 2016;68:343-52.

19. Edwards FH, Cohen DJ, O'Brien SM, et al. Development and validation of a risk prediction model for in-hospital mortality after transcatheter aortic valve replacement. JAMA Cardiol 2016;1:46.

20. Kötting J, Schiller W, Beckmann A, et al. German Aortic Valve Score: a new scoring system for prediction of mortality related to aortic valve procedures in adults. Eur J Cardiothorac Surg 2013;43:971-7.

21. Capodanno D, Barbanti M, Tamburino C, et al. A simple risk tool (the OBSERVANT score) for prediction of 30day mortality after transcatheter aortic valve replacement. Am J Cardiol 2014;113:1851-8.

22. Iung B, Laouénan C, Himbert D, et al. Predictive factors of early mortality after transcatheter aortic valve implantation: individual risk assessment using a simple score. Heart 2014;100:1016-23.

23. Martin GP, Sperrin M, Ludman PF, et al. Novel United Kingdom prognostic model for 30-day mortality following transcatheter aortic valve implantation. Heart 2018;104:1109-16.

24. Seiffert M, Sinning JM, Meyer A, et al. Development of a risk score for outcome after transcatheter aortic valve implantation. Clin Res Cardiol 2014;103:631-40.

25. Debonnaire P, Fusini L, Wolterbeek R, et al. Value of the "tAVI2-SCORe" versus surgical risk scores for prediction of one year mortality in 511 patients who underwent transcatheter aortic valve implantation. Am J Cardiol 2015;115:234-42.

26. Altman DG, Vergouwe Y, Royston P, et al. Prognosis and prognostic research: validating a prognostic model. BMJ 2009;338:b605.

27. Steyerberg EW, Moons KGM, van der Windt DA, et al. Prognosis Research Strategy (PROGRESS) 3: prognostic model research. PLoS Med 2013;10:e1001381.

28. Moons KGM, Kengne AP, Grobbee DE, et al. Risk prediction models: II. External validation, model updating, and impact assessment. Heart 2012;98:691-8.

29. Halkin A, Steinvil A, Witberg G, et al. Mortality prediction following transcatheter aortic valve replacement: A quantitative comparison of risk scores derived from populations treated with either surgical or percutaneous aortic valve replacement. The Israeli TAVR Registry Risk Model Accuracy Assessment study. Int J Cardiol 2016;215:227-31.

30. Pilgrim T, Franzone A, Stortecky S, et al. Predicting mortality after transcatheter aortic valve replacement. Circ Cardiovasc Interv 2017;10:e005481.

31. Arsalan M, Maren W, Hecker F, et al. TAVI risk scoring using established versus new scoring systems: role of the new STS/ACC model. EuroIntervention 2018;13:1520-6.

32. Martin GP, Sperrin M, Hulme W, et al. Relative survival after transcatheter aortic valve implantation: how do patients undergoing transcatheter aortic valve implantation fare relative to the general population? J Am Heart Assoc 2017;6:e007229.

33. Afilalo J, Lauck S, Kim DH, et al. Frailty in older adults undergoing aortic valve replacement: The FRAILTY-AVR Study. J Am Coll Cardiol 2017;70:689-700.

34. Shimura T, Yamamoto $M$, Kano $S$, et al. Impact of the clinical frailty scale on outcomes after transcatheter aortic valve replacement. Circulation 2017;135:2013-24.

35. Kleczynski P, Dziewierz A, Bagienski M, et al. Impact of frailty on mortality after transcatheter aortic valve implantation. Am Heart J 2017;185:52-8.

36. Rogers T, Alraies MC, Pacha HM, et al. Clinical frailty as an outcome predictor after transcatheter aortic valve implantation. Am J Cardiol 2018;121:850-55.

37. Schoenenberger AW, Moser A, Bertschi D, et al. Improvement of risk prediction after transcatheter aortic valve replacement by combining frailty with conventional risk scores. JACC Cardiovasc Interv 2018;11:395-403.

38. Hickey GL, Grant SW, Murphy GJ, et al. Dynamic trends in cardiac surgery: why the logistic EuroSCORE is no longer suitable for contemporary cardiac surgery and implications for future risk models. Eur J Cardiothorac Surg 2013;43:1146-52.

39. Hickey GL, Grant SW, Caiado C, et al. Dynamic prediction modeling approaches for cardiac surgery. Circ Cardiovasc Qual Outcomes 2013;6:649-58.

40. Su TL, Jaki T, Hickey GL, et al. A review of statistical updating methods for clinical prediction models. Stat 
Methods Med Res 2018;27:185-97.

41. Groenwold RHHH, Moons KGMM, Pajouheshnia R, et al. Explicit inclusion of treatment in prognostic modeling was recommended in observational and randomized settings. J Clin Epidemiol 2016;78:90-100.

42. Bagur R, Pibarot P, Otto CM. Importance of the valve durability-life expectancy ratio in selection of a prosthetic aortic valve. Heart 2017;103:1756-59.

43. Arnold SV, Spertus JA, Lei Y, et al. How to define a poor outcome after transcatheter aortic valve replacement: conceptual framework and empirical observations from the placement of aortic transcatheter valve (PARTNER) Trial. Circ Cardiovasc Qual Outcomes 2013;6:591-7.

44. Arnold SV, Reynolds MR, Lei Y, et al. Predictors of poor outcomes after transcatheter aortic valve replacement results from the PARTNER (Placement of Aortic Transcatheter Valve) trial. Circulation 2014;129:2682-90.

45. Arnold SV, Afilalo J, Spertus JA, et al. Prediction of poor outcome after transcatheter aortic valve replacement. J Am
Coll Cardiol 2016;68:1868-77.

46. Fuster V. The proliferation of scoring systems: trying to keep our heads out of the clouds. J Am Coll Cardiol 2017;69:1640-1.

47. Steyerberg EW, Borsboom GJJM, van Houwelingen $\mathrm{HC}$, et al. Validation and updating of predictive logistic regression models: a study on sample size and shrinkage. Stat Med 2004;23:2567-86.

48. Debray TPA, Koffijberg H, Vergouwe Y, et al. Aggregating published prediction models with individual participant data: a comparison of different approaches. Stat Med 2012;31:2697-712.

49. Debray TPA, Koffijberg H, Nieboer D, et al. Metaanalysis and aggregation of multiple published prediction models. Stat Med 2014;33:2341-62.

50. Martin GP, Mamas MA, Peek N, et al. A multiple-model generalisation of updating clinical prediction models. Stat Med 2018;37:1343-58.
Cite this article as: Martin GP, Sperrin M, Mamas MA. Preprocedural risk models for patients undergoing transcatheter aortic valve implantation. J Thorac Dis 2018;10(Suppl 30):S3560S3567. doi: $10.21037 /$ jtd.2018.05.67 